\title{
Isolated Basilar Artery Occlusion Associated with a Clivus Fracture
}

\author{
Abhijit Guha, Mahmood Fazl and Perry W. Cooper
}

\begin{abstract}
Most vascular injuries to the brain secondary to blunt head trauma involve the internal carotid circulation. A case of isolated basilar occlusion secondary to a clival fracture is described and compared to three other cases in the literature.

RÉSUMÉ: Occlusion isolée de l'artère basilaire associée à une fracture du clivus La plupart des blessures vasculaires secondaires à une contusion à la tête impliquent la circulation dans le territoire de la carotide interne. Nous décrivons un cas d'occlusion basilaire isolée secondaire à une fracture du clivus et nous comparons ce cas à trois autres cas décrits dans la littérature.
\end{abstract}

Can. J. Neurol. Sci. 1989: 16:81-83

Trauma to the extracranial or intracranial vessels leading to acute or delayed neurological deficit is well recognized after blunt head injury.' Basilar artery occlusion after trauma has usually been reported as a result of distal thrombus propagation or emboli from an injured vertebral artery.2,3,4 Isolated basilar artery occlusion as a result of blunt head injury associated with a clivus fracture is rare and a literature review revealed only three reported cases.5,6,7 The present case illustrates the clinical course, radiographic findings and outcome of this type of injury and provides further clues to possible mechanisms.

\section{CASE Report}

A previously healthy 27 year old construction worker fell 30 feet landing on his helmet. There was immediate loss of consciousness. At the local hospital his cardiorespiratory status was stable, both pupils were equal $(3 \mathrm{~mm})$ and reactive to light. He responded to pain with extensor posturing on the left and flexion movements on the right. On arrival at Sunnybrook Medical Centre (Regional Trauma Centre) his hemodynamic status was stable and there were attempted spontaneous respiratory movements. There was gross CSF rhinorrhea with no external evidence of a basal skull fracture. The pupils and motor examination remained unchanged and bilateral corneal and gag reflexes were present. Initial axial CT scanning showed opacification of maxillary, ethmoid, and sphenoid sinuses with a small amount of air behind the clivus. There was no intracranial hemorrhage and the brainstem and cerebellum were normal. Bone window images suggested a fracture through the clivus extending to the foramen magnum. A plain lateral cervical film was normal. Richmond bolts for intracranial pressure (ICP) monitoring were inserted bilaterally.

In the Intensive Care Unit, ICP remained stable at $15 \mathrm{mmHg}$ even after cessation of the CSF rhinorrhea over the subsequent 48 hours. Conventional tomography of the skull showed a comminuted fracture of the sella and a clival fracture with a tiny bone fragment near the right occipital condyle. High resolution $\mathrm{CT}$ also demonstrated the fractures (Figure 1) and confirmed the site of fracture of the bony fragment from the edge of the foramen magnum just posterior to the right occipital

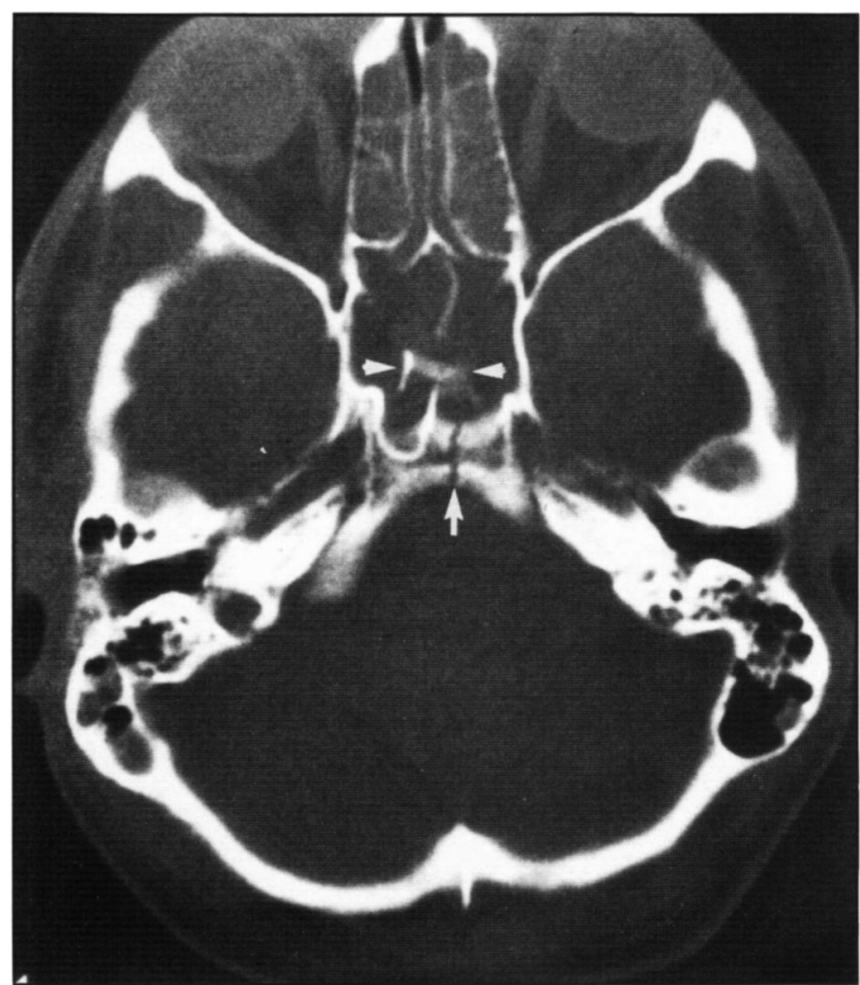

Figure l-Axial CT slice at bone window setting show's a sagittal fracture of the clivus (arrow) and a sellar fracture (arrow heads).

condyle. On day 2 the patient developed tachycardia (110/minute) and hypertension $(210 / 100)$ despite adequate sedation and paralysis. The clinical status remained unchanged over the next five days.

There was deterioration in the neurological status on day 6 with loss of the right corneal reflex, bilateral extensor posturing and "ocular bob-

From the Dept. of Neurosurgery, University of Toronto (A.G.); Div. of Neurosurgery, Sunnybrook Medical Centre, University of Toronto (M.F.); Div. of Neuroradiology, Sunnybrook Medical Centre, University of Toronto (P.W.C.)

Received December 3, 1987. Accepted in final form October 24, 1988

Reprint requests to: M. Fazl, Div. of Neurosurgery, Sunnybrook Medical Centre, University of Toronto, 2075 Bayview Avenue, Ste. A-1038, Toronto, Ontario, Canada M4N 3M5 
bing". A repeat CT scan suggested an early brainstem and right cerebellar infarct. Cerebral angiography on day 7 with selective carotid and vertebral studies showed marked narrowing and occlusion of the basilar artery with a collection of contrast material nearby suggesting a false aneurysm (Figure 2). No early draining veins were present to suggest an arterial-venous fistula. There was no filling of the right anterior inferior cerebellar artery (AICA) and questionable filling of a small left AICA. Carotid injections filled the distal basilar artery just proximal to the superior cerebellar artery (SCA) origins (Figure 3). The left SCA filled well, but on the right only the proximal SCA was faintly filled.

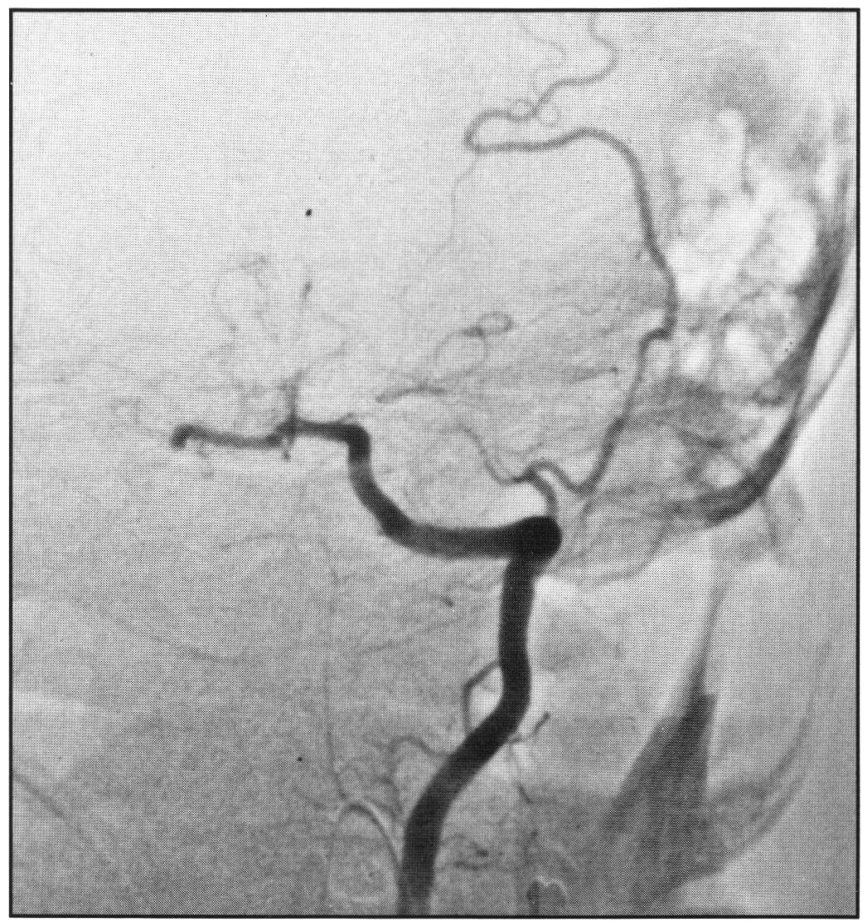

The left posterior inferior cerebellar artery (PICA) did not originate from the left vertebral artery, but filled from the right PICA. Although uncommon this type of filling may be a normal variant. Follow up $\mathrm{CT}$ scans on day 10 and day 36 further delineated the extent of infarction. It included the tectum of the right midbrain, the entire extent of the pons and the superior right cerebellar hemisphere with sparing of the inferior aspect of the cerebellum and the medulla. The grave prognosis precluded any further aggressive management. The patient has remained in a chronic vegetative state.

\section{Discussion}

There are several similarities between our patient and the previously reported cases. ${ }^{5,6,7}$ The mechanism of injury consisted of a massive direct blow to the forehead of vertex resulting in immediate loss of consciousness. There was gross CSF rhinorrhea with blood in the ethmoid and sphenoid sinuses. Transmission of the impact force longitudinally to the atlantooccipital junction resulted in a nondisplaced fracture extending from the sella through the clivus to the foramen magnum. In the previous cases, signs of pontine dysfunction with labile blood pressure, ocular bobbing and absent corneal and gag reflexes appeared almost immediately, whereas in our patient these signs did not develop until about day 6 post injury. This might suggest a different mechanism of basilar occlusion in the present case. The previous patients died 5, 13 and 35 days after injury from cardiorespiratory complications while the present patient is still alive eight months later, though in a vegetative state. $5,6,7$

Autopsy findings in the previous cases confirmed that the mechanism of occlusion was entrapment of the basilar artery in the fracture. The autopsy reported by Anthony et al ${ }^{5}$ showed the basilar artery to be incarcerated within a vertical clivus fracture, from its origin at the vertebral-basilar junction to the origin of the AICA. Infarction of the medical rostral medulla and caudal

Figure 2 - Left vertebral angiogram

a) Towne view shows reflux into distal right vertebral artery with proximal basilar artery narrowing.

b) Lateral view shows collection of contrast medium in mid-clival region suggesting a false aneurysm (arrow heads).

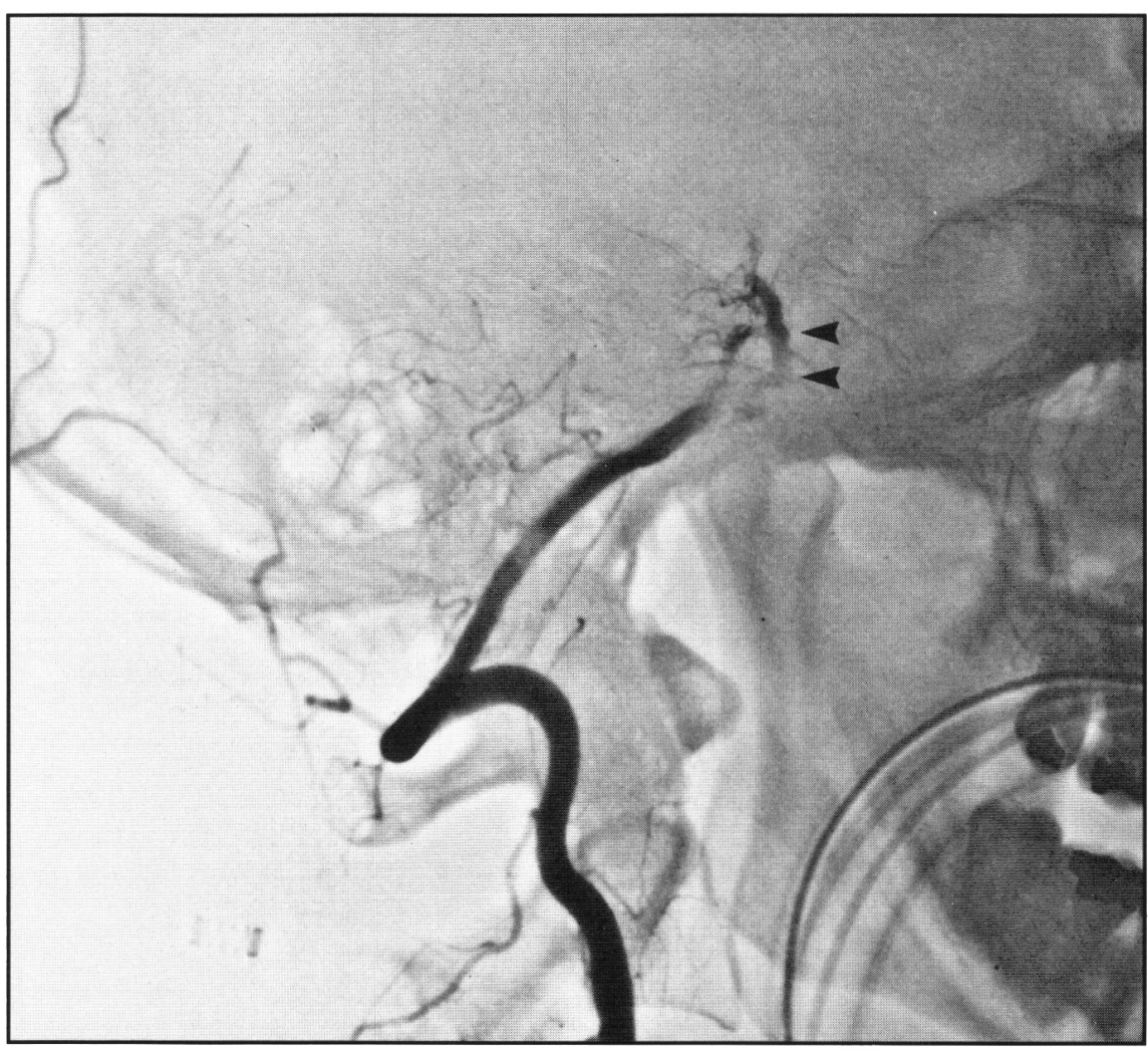




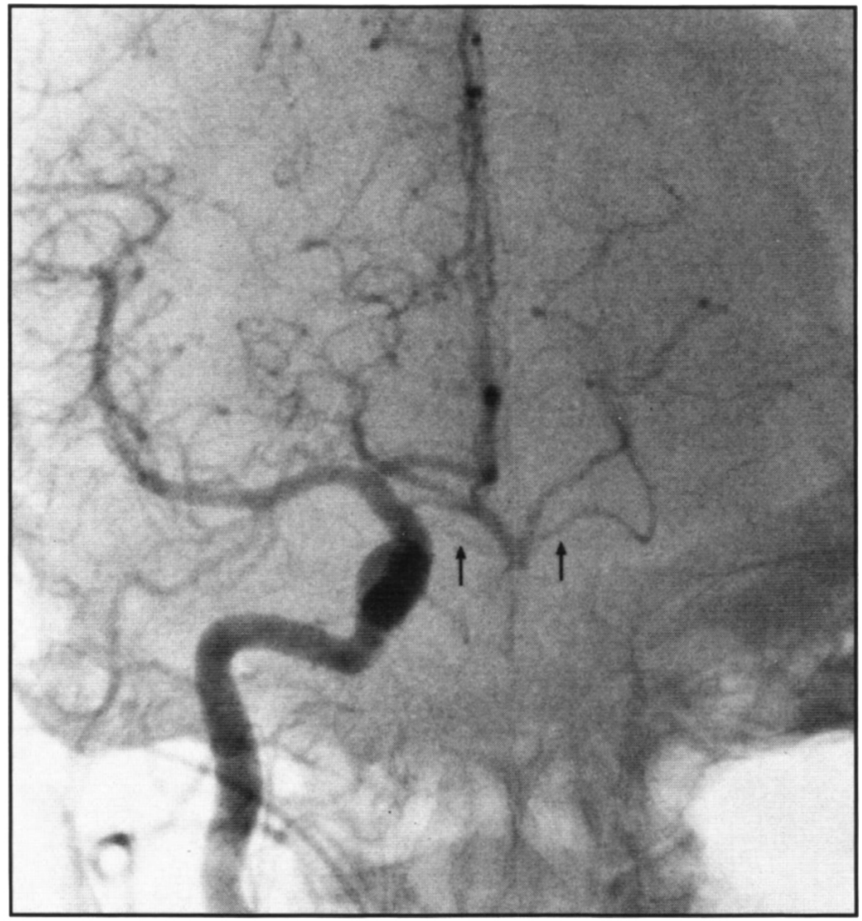

Figure 3 - Right common carotid angiogram fills upper part of the basilar artery. Both superior cerebellar arteries (arrows) are seen. The left is well filled, but only the proximal part of the right is faintly visualized.

pons were the major findings on sectioning. In the case reported by Sights $^{7}$ the entrapment was distal in the origin of the AICA and involved the left SCA leaving the right SCA and PCA patent. Autopsy showed infarction of the pons throughout, more marked in the rostral basal region. There was also infarction of the left cerebral peduncle from its middle portion extending dorsally to involve the inferior colliculi as well as the superior lateral aspect of the left cerebellar hemisphere. In the case reported by Loop et al ${ }^{6}$ both PCA and SCA were spared. However, the patency of the AICA was not confirmed by angiography or autopsy. The autopsy revealed infarction of the entire pons sparing the midbrain, medulla and cerebellum.

Since our patient is still alive, the extent of infarction can only be deduced by the angiographic and CT findings. The angiogram was similar to the previous cases showing sparing of both PCA's along with the vertebral arteries and PICA's. The right SCA filled sparingly in its proximal portion but may have been occluded distally, corresponding to the infarct in the right superior lateral cerebellum and the right tectal region of the midbrain.

The left AICA filled but the right AICA was not seen and was likely occluded. Preservation of the inferior aspects of the cerebellum and the medulla corresponded to the patent vertebral - PICA system. Anomalous filling of the left PICA by the right PICA originating from the right vertebral was also seen.

The crucial nature of blood flow through thalamoperforating branches of the PCA and the paramedian penetrating branches of the basilar artery has been documented in atherosclerotic occlusion of the basilar artery as well as during aneurysmal surgery in this region. 8.9 In all the cases reported the PCA's were spared; occlusion of the paramedian penetrating branches led to infarction of the pons. The delayed clinical picture of pontine dysfunction in our patient does not favour an immediate entrapment, but suggests progressive thrombus formation and occlusion of the penetrating vessels. The initial insult may have resulted in false aneurysm formation or dissection of the basilar artery from intimal damage and subsequent occlusion or embolization of the penetrating pontine vessels. Fractures in this region have also been documented to entrap the vertebral artery leading to lateral medullary infarction or subsequent basilar artery occlusion by extension of thrombus or embolization. ${ }^{7}$

\section{REFERENCES}

1. David JM, Zimmerman RA. Injury of the carotid and vertebral arteries. Neuroradiology 1983; 235: 55-69.

2. Lindenberg R. Incarceration of a vertebral artery in the cleft of a longitudinal fracture of the skull. J. Neurosurg 1966; 24: 908910.

3. Nezami AH, Beremer AM. Basilar artery occlusion in multiple trauma. Case Report. Neurosurgery 1980; 7: 267-270.

4. Woolsey RM, Chung MD. Fatal basilar occlusion following cervical spine injury. Paraplegia 1980; 17: 280-283.

5. Anthony DC, Atwater SK, Rozear MP, et al. Occlusion of the basilar artery within a fracture of the clivus. Case Report J. Neurosurg 1987; 66: 929-931.

6. Loop JW, White LE, Shaw CM. Traumatic occlusion of the basilar artery within a clivus fracture. Radiology $1964 ; 83: 36-40$.

7. Sights WP. Incarceration of the basilar artery in a fracture of the clivus. J. Neurosurg 1968; 28: 588-591.

8. Archer CR, Horenstein S. Basilar artery occlusion, clinical radiological correlation. Stroke 1977; 8: 383-390.

9. Drake CG. Ligation of the vertebral (unilateral or bilateral) or basilar artery in the treatment of large intracranial aneurysms. J Neurosurg 1975; 43: 255-274. 